\title{
RECONSTRUCTION OF THE TRACHEA
}

\author{
BY \\ CHARLES ROB AND L. L. BROMLEY \\ From the Surgical Unit, St. Mary's Hospital, London
}

(RECEIVED FOR PUBLICATION MAY 13, 1953)

Advances in surgical technique and in anaesthesia have made possible a more radical attack on lesions affecting the trachea. Although the number of patients presenting with such a tracheal lesion is small, it is important that they should be treated adequately. Tracheal reconstruction is indicated both for benign and for malignant neoplasms, most of which are found to arise in the lower third. A complete removal may be possible but there is also a place for palliative resection because, untreated, these patients die an unpleasant death from tracheal obstruction. A few tumours are sensitive to irradiation; in these cases there is no place for palliative resection.

\section{Principles of RePair}

The trachea is lined by ciliated columnar epithelium. Its walls contain a rigid framework of cartilaginous rings which are incomplete posteriorly, and it possesses longitudinal elasticity which decreases rapidly during adult life.

In repairing a tracheal defect the rigidity of the walls must be preserved. If a flaccid wall results from operation a flap or flutter develops and impedes deep breathing and coughing: this not only affects respiration adversely but is harmful to the suture line. Moreover, a flaccid wall cannot withstand fibrosis and a stricture may develop. At least one recurrent laryngeal and one superior laryngeal nerve must be preserved, because division of both recurrent laryngeal nerves may obstruct the airway, and division of both superior laryngeal nerves produces an anaesthetic entrance to the larynx so that food and water can be inhaled.

\section{EXPERIMENTAL SURGERY}

Resection and Primary Suture.-Excision of a diseased segment of trachea and end-to-end anastomosis is the best method of repair, as it is for any tube in the body. This is easier in experimental animals than in man. In rats, rabbits, and dogs a considerable length of the trachea can be resected and an anastomosis performed without great tension; mobilization of the lung root increases the mobility of the lower segment.

In four rats we have excised segments of the cervical trachea of four rings, or $7 \mathrm{~mm}$. in length, and performed an anastomosis which has healed satisfactorily without stenosis. The average length of a rat's trachea is $2.8 \mathrm{~cm}$. and so $25 \%$ of the trachea was excised from these animals.

Grindlay, Clagett, and Moersch (1949) report that in the dog the trachea can be anastomosed to the left main bronchus after removal of the right lung, the lower end of the trachea, and the carina. Approximately one-third of the thoracic part of the trachea was removed. The same technique has been applied to man (Grindlay and others, 1949) and two successful reports have been published (Kay, 1952). As a technique, using as it does no prosthesis of foreign material, it has much to recommend it.

RePair of Window Defects.-Many of these can be closed by direct suture and even if the suture line is under a little tension satisfactory healing can be obtained. For defects of moderate size, i.e., not more than $1 \mathrm{~cm}$. in diameter, simple measures will suffice to repair the hole. A free graft of fascia lata (Neuhof, 1917) or a free or pedicled graft of tissue from the neighbourhood, e.g., muscle or pleura, can be used with effect.

For a larger defect rigidity must be provided to support the graft and prevent it from being drawn into the lumen of the trachea and flapping during respiration. Experimental surgery has been carried out using tantalum gauze as a support to a graft (Rob and Bateman, 1949; Swift, Grindlay, and Clagett, 1952). This method was first tried by Rob (1951) in rabbits and sound healing without stenosis was obtained. The fascia lata was placed on the outside of the mesh. This showed that tantalum was inert in the tissues of the neck, that it was strong enough to be used as a support, and that rapid epithelialization developed on the inner or luminal aspect of the graft. Granulation tissue first made its appearance, growing through the mesh, and this was soon covered by epithelium 


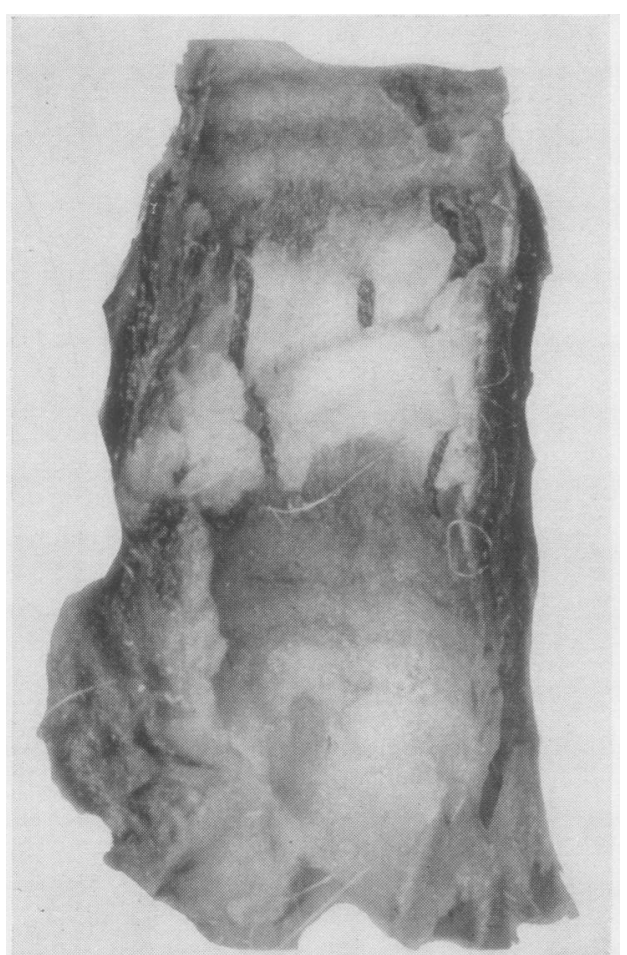

Fig. 1-A rat's trachea two weeks after an autograft had been inserted. The surface epithelium is necrotic. growing inwards from the edges of the graft. This work has been repeated by Swift using dogs; he found the graft to be lined by columnar ciliated epithelium within five or six weeks.

There are certain important points in technique to be noted: (1) The edges of the mesh must be folded so that the sharp ends of wire cannot protrude. (2) The mesh must be moulded to the shape of the trachea. (3) The mesh and fascia lata must overlap the margins of the defect by at least $1 \mathrm{~cm}$. Swift recommended placing the fascia on the inside of the tantalum gauze but we feel that it is better placed on the outside. When on the inside fascia tends to become necrotic and may obstruct the airway.

The use of homologous tracheal grafts, both fresh and preserved, for the repair of small window defects has been reported by Davies, Edmiston, and McCorkle (1952). These grafts are easy to suture into position in dogs. They reported survival of cartilage with epithelialization of the inner aspect of the graft. There may be a place for the use of tracheal grafts for the repair of such defects if small. With larger grafts the expectation of cartilage surviving seems smaller, and if cartilage disintegrates then rigidity, the most important virtue of the graft, is lost.

FIG. 2.-A rat's trachea 143 days after an autograft had been inserted. Some cartilage has survived but most has become necrotic. The size of the lumen has been reduced.

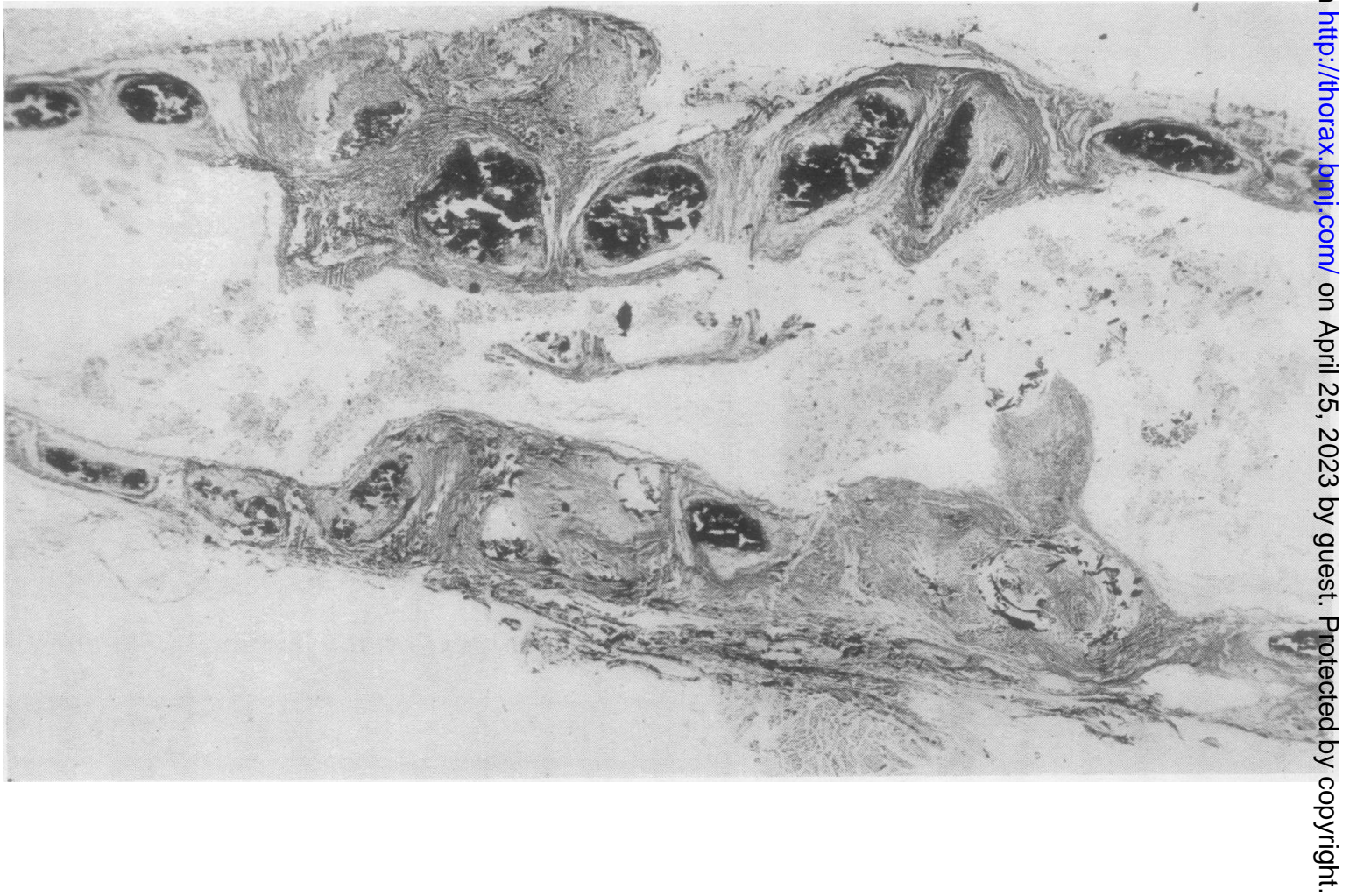


REPair of SegMENTAL DEFECTS.Experimental work has been done to find a satisfactory method of repairing whole defects where a primary anastomosis is impossible.

Tracheal Grafts. -We have experience in rats and rabbits of tracheal grafts, autogenous a n d homologous, both fresh and preserved by freezing in the $\mathrm{manner}$ described for blood vessel grafts (Hufnagel and Eastcott, 1952). These grafts consisted of whole

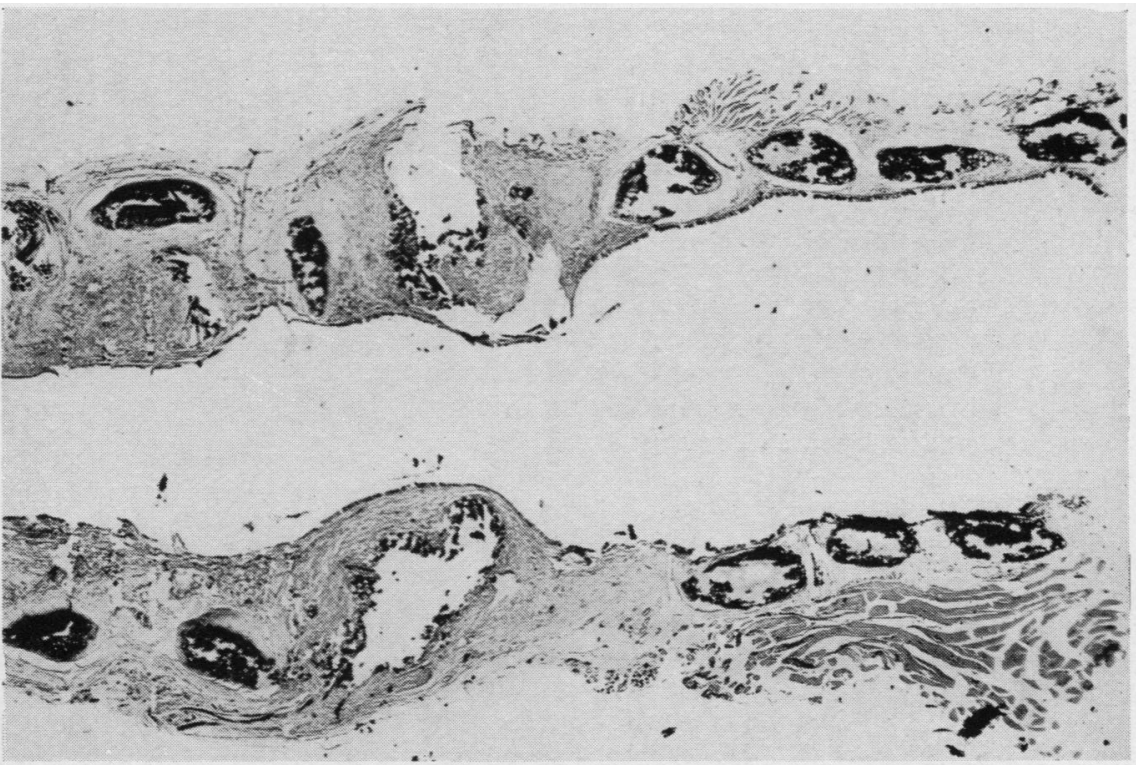

FIG. 3.-A rat's trachea 178 days after a homograft had been inserted. The appearance is similar to that of the autograft in Fig. 1 .

and the closure could usually be made airtight. Certain points will be recorded concerning the subsequent behaviour of these grafts.

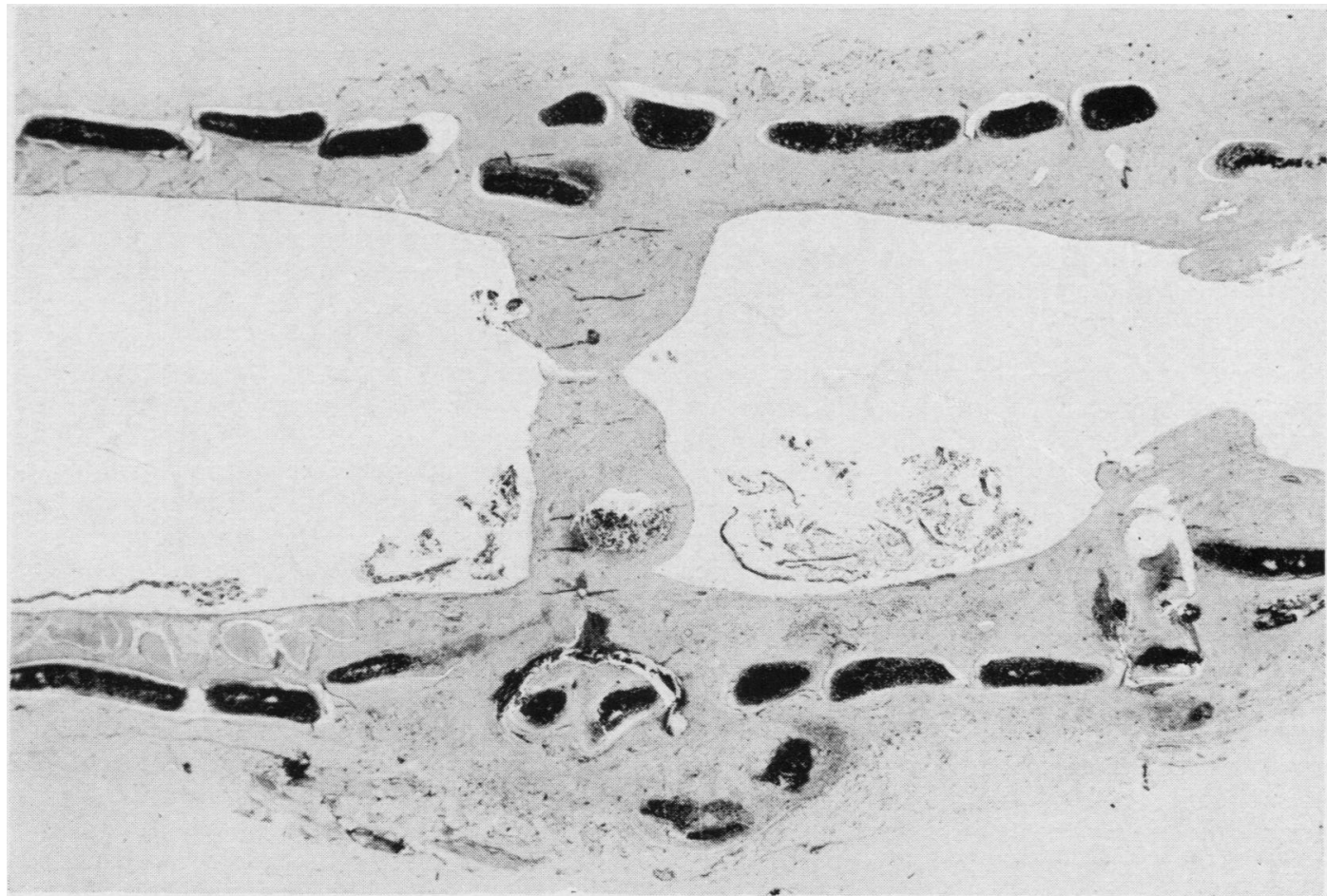

Fig. 4.-Obstruction to the lumen of a rabbit's trachea three weeks after a frozen homograft had been inserted. 
Autographs.-Out of six rats, three developed respiratory obstruction and were sacrificed within 14 days (Fig. 1). In these the graft was found to have sloughed and separated. The other three rats survived longer. One of these had a severe stricture but in the other two, sacrificed at 186 and 200 days, respiration was satisfactory, the external appearance of the trachea was normal, but there was some narrowing of the lumen. In four other rats autografts were inserted in a reversed position, that is with the lower end upwards ; two sloughed but two animals survived. In both these a stricture developed and they were sacrificed after four months.

Microscopically it was found that fibrous tissue replaced the grafted segment and that in several areas cartilage survived but generally it was necrotic (Fig. 2). In the grafts epithelium was reestablished but it was more like squamous metaplasia than normal columnar ciliated epithelium. Thus, if early sloughing and separation of the graft were avoided, the wounds healed and the animals survived for a long time. Nevertheless they all had some degree of stricture. It appears that when cartilage only survives partially it is inadequate to maintain rigidity of the graft.

Homografts.-In these we had experiences similar to those with autografts. Eight rats were used. In five the graft separated within two weeks. In two survivors severe stenosis developed and these were sacrificed after 104 and 130 days. The remaining animal, which was killed after 175 days, had only minimal narrowing of the lumen. Microscopic examination again showed poor survival of cartilage (Fig. 3). The graft was replaced by fibrous tissue and was lined with a more flattened columnar epithelium.

Frozen Homografts.-The results of the use of frozen grafts were disappointing. In 10 rats not one survived for longer than 21 days. In all there was rapid sloughing and separation of the graft. In the animal which survived longest (21 days), before being killed for respiratory difficulty, the cartilage of the graft was necrotic. In three rabbits frozen homografts failed. In one animal the graft separated within three days ; in the other two strictures developed and these animals were sacrificed after three weeks. Fig. 4 shows the cartilage surviving, but the cells do not appear normal as some central disintegration of the pattern is apparent.

From these experiments it appears that homografts, whether fresh or preserved, are unlikely to be of value in the repair of tracheal defects.
Whether cartilage will survive is doubtful, and it has been our experience that stricture formation is almost certain. It appears that some rigid foreign material should be used in conjunction with a homograft if the latter is to be used at all.

Polythene Tubes or Vitellium Tubes.-The use of these tubes gives good results in experimental animals. Of the two materials, polythene is to be preferred as it is unwettable, thus mucoid secretions will not stick to it and block the lumen. The main difficulty is to find a satisfactory method of fixing the tube so that it remains firmly anchored at each end. It is found that a new epithelium grows on the outside of these tubes, and so workers were encouraged to remove the tube endoscopically when healing was considered to be complete. When the tube was removed it left a fibrous wall to the trachea with an epithelial lining, but soon afterwards the lumen narrowed from contraction of the fibrous tissue. It seems certain that if a tube can be anchored in position it should remain permanently, hence the necessity for finding a method whereby the tube can be anchored firmly. If the tube should slip, even a little, a stricture develops in the small unsupported zone above the tube.

Cotton and Penido (1952) reported two cases in man where a steel tube had been used with success but where difficulties were encountered due to the tube slipping.

Fascia Reinforced with Tantalum or StainLESS STEel Wire.-In many instances where a tracheal tumour has been resected it may be possible to leave a bridge of epithelium uniting the upper and lower ends, and the presence of such a bridge, however narrow, will facilitate the reconstructive procedure. Belsey (1946) advocated and used successfully a coil of stainless steel wire reinforced with fascia. In one of his cases he built a new segment 3 in. long in the intrathoracic trachea after removing an adenocarcinoma. His patient survived two and a half years before dying of a recurrence.

Tantalum gauze with a fascial wrapping can be used to repair large defects, and the value of having a bridge of epithelium so that the gap is not complete has been stressed. Rob and Bateman (1949) employed this method after excising a recurrence of a thyroid carcinoma which was infiltrating the trachea. The patient is alive and well five years after operation and there is no stenosis on bronchoscopy. We have used this method with success in four other patients, but they have all died within one year from metastases. 
Cahan (1952) reported a case where tantalum and fascia were used successfully to repair a large defect in the lower third of the trachea after removal of an adenocarcinoma. A small bridge of trachea was left behind.

\section{CONCLUSIONS}

In the rat tracheal grafts, both autogenous and homologous, fail to provide a satisfactory method for reconstruction. Many become necrotic and separate. The survivors develop a stricture. Homografts preserved by freezing are more unsatisfactory. Direct suture under considerable tension provides a satisfactory repair.

For large defects a prosthesis is necessary. A mesh of steel or tantalum wire covered with fascia lata has been satisfactory for this purpose. A patient whose trachea was reconstructed in this way has now been asymptomatic for over five years.

\section{REFERENCES}

Belsey, R. (1946). Thorax, 1, 39.

Cahan, W. G. (1952). J. thorac. Surg., 23, 513.

Cotton, B. H., and Penido, J. R. F. (1952). Ibid., 24, 231.

Davies, O. G., Edmiston, J. M., and McCorkle, H. J. (1952). Ibid., 23, 367.

Grindlay, J. H., Clagett, O. T., and Moersch, H. J. (1949). Proc. Mayo Clinic, 24, 555.

Hufnagel, C. A., and Eastcott, H. H. G. (1952). Lancet, 1, 531.

Kay, E. B. (1952). J. thorac. Surg., 24, 243.

Neuhof, H. (1917). Surg. Gynec. Obstet., 24, 383.

Rob, C. G. (1951). In Surgical Progress, 1951, ed. Carling, E. Rock, and Ross, J. Paterson, 194. Butterworth, London.

and Bateman, G. H. (1949). Brit. J. Surg., 37, 202.

Swift, E. A., Grindlay, J. H., and Clagett, O. T. (1952). J. thorac. Surg., 24, 482. 